\title{
Chemistry induced by energetic ions in water ice mixed with molecular nitrogen and oxygen
}

\author{
Ph. Boduch ${ }^{1}$, A. Domaracka ${ }^{1}$, D. Fulvio ${ }^{2}$, T. Langlinay ${ }^{1}$, X. Y. Lv ${ }^{1}$, M. E. Palumbo ${ }^{3}$, H. Rothard ${ }^{1}$, and G. Strazzulla ${ }^{3}$ \\ ${ }^{1}$ Centre de Recherche sur les Ions, les Matériaux et la Photonique, CIMAP - CIRIL - GANIL (CEA/CNRS/ENSICAEN/ \\ Université de Caen-Basse Normandie), boulevard Henri Becquerel, BP 5133, 14070 Caen Cedex 05, France \\ 2 Laboratory for Atomic and Surface Physics, University of Virginia, 351 McCormick Road, PO Box 400238, Charlottesville, \\ VA 22904-4238, USA \\ 3 INAF - Osservatorio Astrofisico di Catania, via S. Sofia 78, 95123 Catania, Italy \\ e-mail: gianni@oact.inaf.it
}

Received 7 April 2012 / Accepted 16 June 2012

\begin{abstract}
Context. Several molecular species have been observed as frozen gases in cold environments such as grains in the interstellar/circumstellar medium or icy objects in the outer solar system. Because $\mathrm{N}_{2}$ and $\mathrm{O}_{2}$ are homonuclear, symmetric molecules are not easily observed. It is therefore relevant to find indirect methods to prove their presence from astronomical observations. Aims. Here we investigate one of the possible indirect methods, namely the formation of specific molecules by cosmic ion bombardment of ices in astrophysical environments that contain $\mathrm{O}_{2}$ and $\mathrm{N}_{2}$. The observation of these molecules in astronomical environments could act as a trojan horse to detect the presence of frozen molecular oxygen and/or nitrogen.

Methods. We have conducted ion bombardment experiments of frozen $\mathrm{O}_{2}, \mathrm{H}_{2} \mathrm{O}$ and their mixtures with $\mathrm{N}_{2}$ at the laboratories of CIMAP-GANIL at Caen (France) and LASp at Catania (Italy). Different ions $\left({ }^{13} \mathrm{C}^{2+}, \mathrm{Ar}^{2+}\right.$ and $\mathrm{H}^{+}$) and energies (30-200 keV) have been used.

Results. We have found that ${ }^{13} \mathrm{CO}_{2}$ is formed when carbon ions are implanted in ices containing $\mathrm{H}_{2} \mathrm{O}$ and/or $\mathrm{O}_{2}$. Ozone and nitrogen oxides ( $\mathrm{NO}, \mathrm{N}_{2} \mathrm{O}, \mathrm{NO}_{2}$ ) are formed in the studied ices containing $\mathrm{O}_{2}$ and $\mathrm{N}_{2}$ with different relative abundances.

Conclusions. We suggest that ozone and nitrogen oxides are present and have to be searched for in some specific environments such as dense clouds in the interstellar medium and the surfaces of Pluto, Charon and Triton. Their observation could demonstrate the presence of molecular oxygen and/or nitrogen. A possible interest for the observations of atmospheres in exo-planetary objects is also discussed.
\end{abstract}

Key words. astrochemistry - radiation mechanisms: non-thermal - techniques: spectroscopic - minor planets, asteroids: general dust, extinction

\section{Introduction}

More than 160 molecular species have been detected in the gas phase in space ${ }^{1}$. Less than 20 are observed frozen on the surface of so-called icy bodies in the solar system and on the interstellar grains in dense molecular clouds. Molecular clouds are the site of star formation and complex organic molecules formed in these regions are thought to be incorporated in planets; satellites and comets also formed with a new star. One open question is related to the origin of molecules in dense molecular clouds. Based on our present knowledge, most diatomic species (an important exception being $\mathrm{H}_{2}$ ) are formed in the gas phase while the origin of complex molecules is still debated. It is generally accepted that many complex molecules form in the solid phase in icy grain mantles (e.g. Fuchs et al. 2009). The presence of icy mantles is clearly demonstrated by infrared observations (e.g., Willner et al. 1982; Whittet et al. 1988; Smith et al. 1989; Tielens et al. 1991; Gerakines et al. 1999; Gibb et al. 2000; Boogert et al. 2004; Bergin et al. 2005). Once formed, icy grain mantles are exposed to the interaction with low-energy cosmic ions (keV-MeV) and UV photons (6.2-13.6 eV). When cosmic ions penetrate icy grain mantles, they release their energy mainly

\footnotetext{
${ }^{1}$ http://astrochymist.org/astrochymist_ism.html
}

through ionizations and excitations of target molecules along the ion track. As a consequence, molecular bonds are broken and molecular fragments recombine, giving rise to molecular species not present in the original mantle (e.g., Palumbo \& Strazzulla 1993; Palumbo et al. 1998; Pilling et al. 2010). Species more volatile and less volatile with respect to the original ones are formed. If C-bearing species are present in the original mantle, a refractory residue is left over after warm-up to the sublimation temperature of volatile species (e.g. Moore et al. 1983; Foti et al. 1984; Strazzulla \& Baratta 1992; Palumbo et al. 2004). Because $\mathrm{N}_{2}$ and $\mathrm{O}_{2}$ are homonuclear, symmetric molecules are not easily observed, although very low intensity IR bands can be activated in the solid phase due to the perturbations of neighboring molecules (e.g. Ehrenfreund et al. 1992). It is therefore relevant to find indirect methods to prove, from astronomical observations, their presence in cold environments such as grains in the interstellar/circumstellar medium or icy objects in the outer solar system. Indeed, nitrogen and oxygen are expected to freeze out onto icy grain mantles in dense molecular clouds (e.g. Caselli et al. 2002; Bergin et al. 2002). The astronomical observations we refer to are not only those relative to solid-state infrared (IR) absorption bands, but also gas-phase at radio or visible wavelengths or even mass spectroscopy (e.g. for "in situ" observations of comets) after sublimation of the ices. 
Water ice is widely present, often as the most abundant chemical species, on the surfaces of the majority of the airless objects in the solar system. Many other molecules have also been identified or are suspected to be present (for a recent review see Dalton et al. 2010). Concerning the molecules of interest to the present paper, $\mathrm{O}_{2}$ has been observed on Europa, Callisto, and Ganymede (Spencer \& Calvin 2002), possibly produced, along with other oxidants such as $\mathrm{H}_{2} \mathrm{O}_{2}$ and $\mathrm{O}_{3}$, by radiolysis of water-ice and/or its mixtures with other species (e.g. $\mathrm{CO}_{2}$, e.g. Boduch et al. 2011). $\mathrm{O}_{3}$ has also been observed in the atmosphere of Dione and Rhea, possibly as a consequence of its release from radiolysed surfaces (Johnson et al. 2008). $\mathrm{N}_{2}$ has been firmly identified on Pluto (Owen et al. 1993; Douté et al. 1999) and Triton (Cruikshank et al. 1993). It could be present also on Charon and, almost certainly, on some trans-Neptunian objects.

In the interstellar medium, the observed solid-state IR absorption bands are compatible with two different scenarios: in the first one $\mathrm{H}_{2} \mathrm{O}$-rich mantles form e.g. during the contraction of the cloud. When the density increases to more than about $10^{4} \mathrm{~cm}^{-3}$, CO-rich mantles would accrete on the top. On the other hand, two different grain populations could exist: the first one with CO-rich (mixed with non-polar species) mantles in the inner and denser portion of the cloud; the second population, with $\mathrm{H}_{2} \mathrm{O}$-dominated mantles, would prevail in the outer portion of the cloud. In both cases it is expected that $\mathrm{O}_{2}$ and $\mathrm{N}_{2}$ can also accrete onto icy mantles.

For these reasons it is interesting to study the spectral properties of ice mixtures containing $\mathrm{O}_{2}$ and $\mathrm{N}_{2}$ and the chemistry induced by ion bombardment experiments simulating the effects of cosmic ion bombardment. In this paper we present results obtained by irradiating frozen $\mathrm{O}_{2}, \mathrm{H}_{2} \mathrm{O}$ and their mixtures with $\mathrm{N}_{2}$ with the aims: (1) to provide an indirect evidence for $\mathrm{N}_{2}$ on $\mathrm{O}_{2}$ in ices and (2) to investigate ion-irradiation chemistry for mixtures not considered before.

\section{Experimental}

Most of the experiments have been performed at ARIBE, the low-energy beam facility of GANIL (Grand Accélerateur National d'Ions Lourds, Caen, France). Pure or mixed gases were deposited at low temperature $(15 \mathrm{~K})$ onto IR-transparent substrates (CsI) in a high-vacuum chamber $\left(P \leq 10^{-7} \mathrm{mbar}\right)$. The chamber is connected through IR-transparent windows, to a Fourier transform infrared (FTIR) spectrophotometer (Nicolet Magna 500). More details can be found elsewhere (e.g. Pilling et al. 2010). The sample-cryostat system can be rotated and fixed at three different positions to allow gas deposition, FTIR measurement and perpendicular ion bombardment.

The ions are produced in a $14.5 \mathrm{GHz}$ electron cyclotron resonance (ECR) ion source, and are then extracted by a voltage of $U=15 \mathrm{kV}$. The energy of ions with charge $q$ is $E=q U$. After selection of mass and charge by a dipole magnet, $30 \mathrm{keV}$ ${ }^{13} \mathrm{C}^{2+}$ ions are guided toward the collision chamber. The use of isotopically labeled carbon ions allows a much more accurate determination of the yields of production of new species that contain the projectile, carbon mono and di-oxide in the present case. Indeed, ${ }^{12} \mathrm{CO}_{2}$ is present as residual gas along the path of the IR beam and its bands often appear in the collected spectra. The ion beam is swept horizontally and vertically by a device that consists of two sets of parallel plates to ensure a uniform irradiation of the target on an area of about $1 \times 1 \mathrm{~cm}^{2}$, i.e. more extended than the area probed by the IR beam. An insulated diaphragm is placed between the sweeping device and the target.
Table 1. List of the experiments.

\begin{tabular}{lcc}
\hline \hline Target & $\begin{array}{c}\text { Beam } \\
\text { (energy in keV) }\end{array}$ & Laboratory \\
\hline $\mathrm{H}_{2} \mathrm{O}$ & ${ }^{13} \mathrm{C}^{2+}(30 \mathrm{keV})$ & CIMAP-GANIL-Caen \\
$\mathrm{H}_{2} \mathrm{O}: \mathrm{N}_{2}: \mathrm{O}_{2}$ & ${ }^{13} \mathrm{C}^{2+}(30 \mathrm{keV})$ & CIMAP-GANIL-Caen \\
$\mathrm{N}_{2}: \mathrm{O}_{2}$ & ${ }^{13} \mathrm{C}^{2+}(30 \mathrm{keV})$ & CIMAP-GANIL-Caen \\
\hline $\mathrm{H}_{2} \mathrm{O}: \mathrm{N}_{2}$ & $\mathrm{Ar}^{2+}(60 \mathrm{keV})$ & LASp-Catania \\
$\mathrm{O}_{2}$ & $\mathrm{H}^{+}(200 \mathrm{keV})$ & LASp-Catania \\
\hline
\end{tabular}

A Faraday-cup can be inserted in front of the target. In this condition, the currents on the diaphragm $\left(I_{\mathrm{D}}\right)$ and in the Faraday-cup $\left(I_{\mathrm{CF}}\right)$ are measured by means of current integrators before each step of ion bombardment of the target. Then the ratio of $I_{\mathrm{CF}} / I_{\mathrm{D}}$ is determined. This procedure allows one to know the current and thus the flux on the target by measuring the diaghragm current $I_{\mathrm{D}}{ }^{\prime}$ during ion bombardment, when the Faraday-cup is removed to expose the target to the beam. The ratio is assumed to be constant during each step of ion bombardment.

The thicknesses of the samples (2-10 micrometers, assuming an ice density of $1 \mathrm{~g} / \mathrm{cm}^{3}$ ) were much larger than the penetration depth (2000 and $4000 \AA)$ of the used ions $\left(30 \mathrm{keV}{ }^{13} \mathrm{C}^{2+}\right.$ ) that are then "implanted" in the target. The ion current density was maintained to be sufficiently low to avoid a macroscopic heating of the samples. The spectra were taken with a resolution of $1 \mathrm{~cm}^{-1}$.

Some additional experiments have been performed at the Laboratorio di Astrofisica Sperimentale (LASp) of INAF Osservatorio Astrofisico di Catania (Italy). The experimental setup is composed of a stainless steel high-vacuum chamber $\left(P<10^{-7}\right.$ mbar) interfaced to an ion implanter (Danfysik 1080-200; for details on the experimental apparatus see Strazzulla et al. 2001). The sample is mounted at an angle of $45^{\circ}$ with respect to both the ion beam and the infrared beam of an FTIR spectrophotometer (Bruker Equinox 55) faced to the vacuum chamber through potassium bromide $(\mathrm{KBr})$ windows. This experimental set-up enable us to acquire "in-situ" spectra before and after each step of ion bombardment, without tilting or rotating the sample. The irradiated samples were pure $\mathrm{O}_{2}$ and mixtures $\mathrm{H}_{2} \mathrm{O}: \mathrm{N}_{2}$. The thicknesses of the samples studied in Catania were thinner than the penetration depth of the used ions $\left(60 \mathrm{keV} \mathrm{Ar}^{++}\right.$or $\left.200 \mathrm{keV} \mathrm{H}^{+}\right)$, which then deposit only a fraction of their energy in the ice and are stopped in the substrate (a silicon wafer). Here the ion current density was also maintained sufficiently low to avoid a macroscopic heating of the samples. The spectra were taken with a resolution of $1 \mathrm{~cm}^{-1}$. The experiments with thin films are more appropriate to simulate the effects of cosmic ions bombarding interstellar grains; implantation experiments better simulate the effects induced by, e.g., solar wind and magnetospheric ions bombarding icy surfaces of objects in the solar system. A list of the experiments discussed in this paper is given in Table 1 .

The energy lost by ions that interact with the target is redistributed among the present species and causes displacements of atoms from their lattice position and breaking of molecular bonds whose recombination may lead to the production of new species. In our experiments we used some reactive ions that, as discussed many times in the literature (for a review see Strazzulla, 2011), induce the same effect as any other ion, but in addition have a chance to form new species containing the projectile. Each incoming ion with energy of several keV breaks thousands of bonds and many new molecules can be formed per single incoming ion. On the other hand, a maximum 
$\mathrm{Ph}$. Boduch et al.: Energetic ions induced chemistry

Table 2. List of the newly formed molecules after implantation of $30 \mathrm{keV}^{13} \mathrm{C}^{2+}$ ions in different ice mixtures (see Fig. 1).

\begin{tabular}{lccc}
\hline \hline Species & $\begin{array}{c}\text { Peak position } \\
\mathrm{cm}^{-1}\end{array}$ & $\begin{array}{c}\text { Band strengths } \\
\text { cm molecule }\end{array}$ & References \\
\hline${ }^{13} \mathrm{CO}_{2}$ & 2277 & $7.6 \times 10^{-17}$ & Yamada \& Person (1964) \\
$\mathrm{N}_{2} \mathrm{O}$ & 2234 & $1.07 \times 10^{-17}$ & Fulvio et al. (2009) \\
${ }^{13} \mathrm{CO}$ & 2092 & $1.1 \times 10^{-17}$ & Jiang et al. (1975), Loeffler et al. (2005) \\
$\mathrm{NO}$ & 1870 & $4.5 \times 10^{-18}$ & Pugh \& Rao (1976), Sicilia et al. (2012) \\
$\mathrm{NO}_{2}$ & 1605 & $6.24 \times 10^{-18}$ & Fulvio et al. (2009) \\
$\mathrm{O}_{3}$ & 1038 & $1.4 \times 10^{-17}$ & Smith et al. (1985) \\
\hline
\end{tabular}

Notes. Peak positions of the bands used to evaluate their column densities and the band strength are also given.

yield of one molecule containing the projectile (e.g. ${ }^{13} \mathrm{CO}_{2}$ in the present experiments) can be obtained. The column densities (molecules $\mathrm{cm}^{-2}$ ) of newly synthesized species were calculated by dividing the area $\left(\mathrm{cm}^{-1}\right)$ of a given band (measured in an optical depth scale) by its band strength (cm molecule $\left.{ }^{-1}\right)$. The band area was calculated by removing the underlying continuum. It is relevant to say that the uncertainty on the values of the band strengths is high (20-30\%) and thus the same is true for error on abundance derivation. In Table 2, the peak positions of the band used to evaluate the column density of a given species are reported together with the band strength values. We assumed that the values for the band strengths for different isotopically labeled species are the same.

\section{Results}

Absorbance IR spectra of different mixtures that are deposited at $15 \mathrm{~K}$ and after they were irradiated by $30 \mathrm{keV}{ }^{13} \mathrm{C}^{2+}$ ions are shown in Fig. 1. The continuum is due to the interference pattern of the IR beam that passes through the icy film. The thicknesses of the ice mixtures (2-10 micrometers) are always much greater than the penetration depth of the bombarding ions that are then implanted in the sample. The penetration depths of ${ }^{13} \mathrm{C}^{2+}$ in the samples ranges between 2000 and $4000 \AA$. It is not easy to determine which are the exact fractions of each component in the mixtures. This is connected with the difference in the sublimation temperatures of the $\mathrm{H}_{2} \mathrm{O}, \mathrm{N}_{2}$ and $\mathrm{O}_{2}$. Therefore, the molecular fractions that we prepared in the gas phase before admitting them into the vacuum chamber are different from the fractions that are really deposited on the substrate.

Since $\mathrm{N}_{2}$ and $\mathrm{O}_{2}$ do not exhibit any band in the IR spectrum, the only features caused by the deposited mixtures are those of water ice. In the shown spectral range water has only a broad band at about $2200 \mathrm{~cm}^{-1}$. In the spectra of implanted mixtures features appear through the formation of species that are originally not present (Fig. 1). These include both species that contain the implanted projectile and species that contain only atoms present in the original target (see Table 2).

The column densities of ${ }^{13} \mathrm{CO}_{2}$ and ${ }^{13} \mathrm{CO}$ formed after implantation of the carbon ions in different ice mixtures are reported in Fig. 2. We see that

- Pure $\mathrm{H}_{2} \mathrm{O}$ : only ${ }^{13} \mathrm{CO}_{2}$ forms. It scales almost linearly with the ion fluence ( $Y=0.57 \mathrm{~mol} / \mathrm{ion})$. We also have conducted other implantation experiments with multiply charged carbon ions in water ice that will be published in a different paper (Lv et al., in prep.).

- $\mathrm{H}_{2} \mathrm{O}: \mathrm{N}_{2}: \mathrm{O}_{2}$ : both ${ }^{13} \mathrm{CO}_{2}$ and ${ }^{13} \mathrm{CO}$ form. The yield of formation of carbon dioxide is equal, within errors, to the case of pure water ice. The yield for carbon monoxide is about $Y=0.25 \mathrm{~mol} /$ ion.

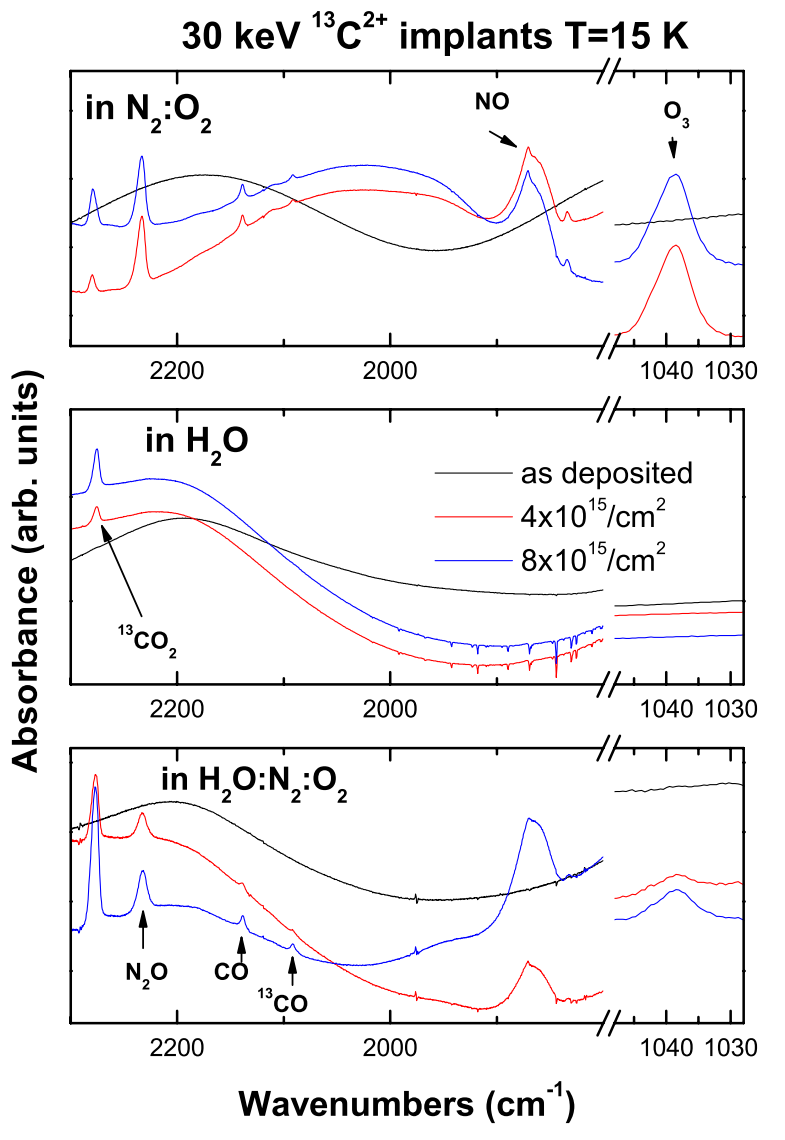

Fig. 1. Absorbance IR spectra of different mixtures as deposited at $15 \mathrm{~K}$ (continuum featureless black lines) and after having been irradiated by $30 \mathrm{keV}{ }^{13} \mathrm{C}^{2+}$ ions (at two different ion fluences, red and blue lines). The continuum is due to the interference pattern of the IR beam that pass through the icy film. (Note the appearance of ${ }^{12} \mathrm{CO}$ is due to ion bombardment of ${ }^{12} \mathrm{CO}_{2}$ that is present as a contaminant (not shown).)

- $\mathrm{N}_{2}: \mathrm{O}_{2}$ : both ${ }^{13} \mathrm{CO}_{2}$ and ${ }^{13} \mathrm{CO}$ form. At low implantation ion fluence, carbon monoxide is more abundant than carbon dioxide.

This is better clarified in Fig. 3 where the ${ }^{13} \mathrm{CO}_{2} /{ }^{13} \mathrm{CO}$ column density ratio is shown. Please note that for pure water ice ${ }^{13} \mathrm{CO}$ is below the detection limit at the ion fluences used in these experiments and therefore we cannot measure that ratio. No error bars are shown in the figures because they are much lower than the systematic errors due to the error in the band strengths that, as said, is about 20-30\%. The data indicate that

(1) The formation of ${ }^{13} \mathrm{CO}_{2}$ in pure $\mathrm{H}_{2} \mathrm{O}$ is dominated by these reactions:

$\mathrm{H}_{2} \mathrm{O} \rightarrow \mathrm{H}+\mathrm{OH}$ 

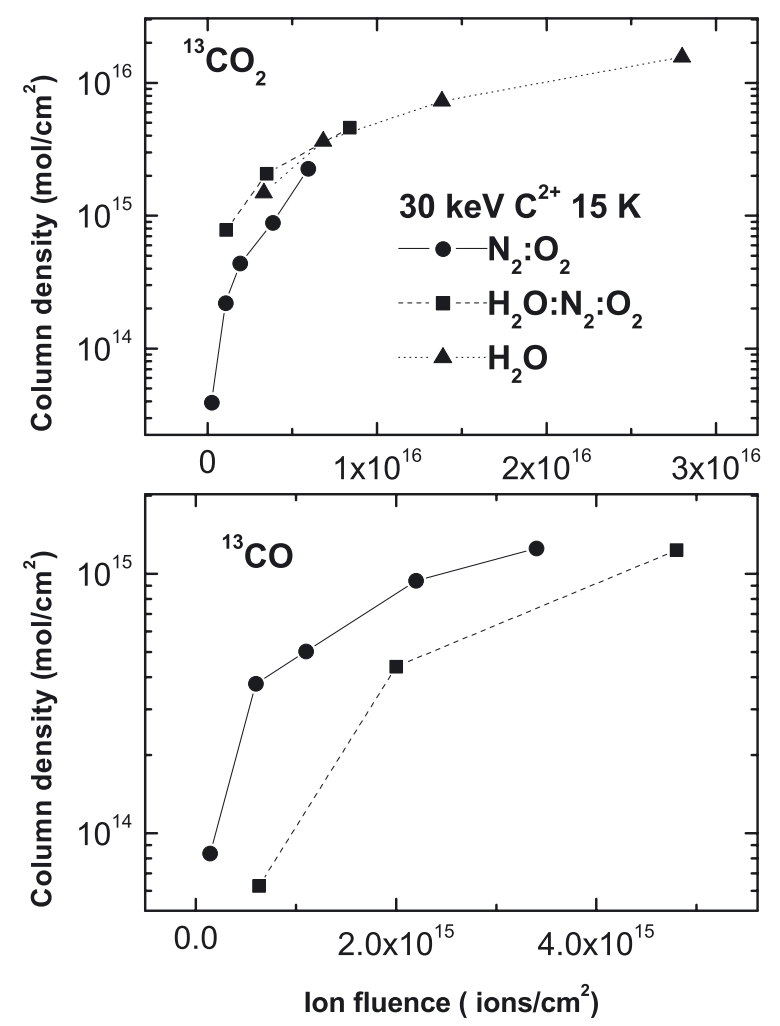

Fig. 2. Column density of ${ }^{13} \mathrm{CO}_{2}$ and ${ }^{13} \mathrm{CO}$ formed after implantation of $30 \mathrm{keV}^{13} \mathrm{C}^{2+}$ ions in different ice mixtures at $15 \mathrm{~K}$.

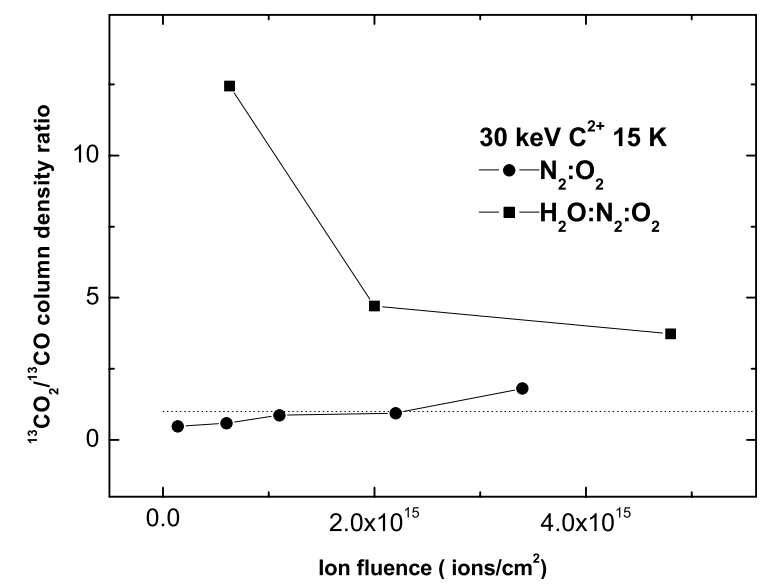

Fig. 3. ${ }^{13} \mathrm{CO}_{2} /{ }^{13} \mathrm{CO}$ column density ratio of ice mixtures irradiated with $30 \mathrm{keV}{ }^{13} \mathrm{C}^{2+}$ ions. A straight line corresponding to a ratio equal to 1 is depicted to guide the eye.

$$
\begin{aligned}
& \mathrm{C}+\mathrm{OH} \rightarrow \mathrm{HCO} \\
& \mathrm{HCO}+\mathrm{OH} \rightarrow \mathrm{CO}_{2}+\mathrm{H}_{2} .
\end{aligned}
$$

(2) When $\mathrm{O}_{2}$ is the only oxygen-bearing molecule (i.e. in the $\mathrm{O}_{2}: \mathrm{N}_{2}$ mixture), $\mathrm{CO}_{2}$ is formed from $\mathrm{CO}$ :

$$
\begin{aligned}
& \mathrm{O}_{2}+\mathrm{C} \rightarrow \mathrm{CO}+\mathrm{O} \\
& \mathrm{CO}+\mathrm{O} \rightarrow \mathrm{CO}_{2} .
\end{aligned}
$$

We have also searched for other species that could be formed after carbon implantation but we have not detected other C-bearing species. This might be because the amount of implanted carbon is not sufficient to produce other species in detectable quantity.

Column densities of nitrogen oxides and ozone formed after implantation of $30 \mathrm{keV}{ }^{13} \mathrm{C}^{2+}$ ions in different ices are reported

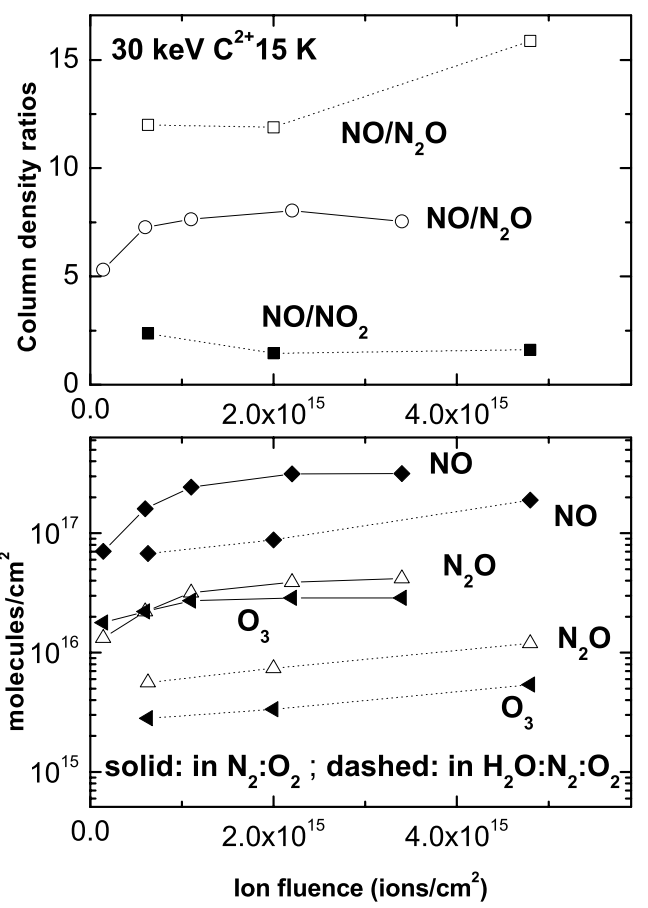

Fig. 4. Results relative to nitrogen oxides and ozone formed after implantation of $30 \mathrm{keV}^{13} \mathrm{C}^{2+}$ ions in different ices. Points connected by solid lines refer to the $\mathrm{N}_{2}: \mathrm{O}_{2}$ mixture, those connected by dashed lines refer to $\mathrm{H}_{2} \mathrm{O}: \mathrm{N}_{2}: \mathrm{O}_{2}$ mixtures. Top panel: column density ratios of nitrogen oxides. Bottom panel: column density of nitrogen oxides and ozone.

in Fig. 4 (bottom panel). It is interesting to note that the original $\mathrm{N}_{2}$ and $\mathrm{O}_{2}$ are not detectable in the ice mixtures prepared as described previously, their presence is made clear only after ion bombardment by the formation of nitrogen oxides and/or ozone. It is important to note that the column densities of the nitrous species presented in Fig. 4 are, on average, at least one order of magnitude higher than those of the species that contain the implanted projectile (Fig. 2). This is because reactive ions (e.g., $\mathrm{H}^{+}, \mathrm{C}^{+}, \mathrm{N}^{+}, \mathrm{O}^{+}, \mathrm{S}^{+}$) induce all effects of any other ion, but in addition have a chance, by implantation in the target, to form new species that contain the projectile (Strazzulla 2011). Each incoming ion with energies between 10 and $200 \mathrm{keV}(30 \mathrm{keV}$ carbon in the present experiments) breaks $10^{3}-10^{4}$ bonds and many new molecules can be formed per single incoming ion by recombination of fragments of the irradiated species. In addition, only reactive ions have a chance to form species that include the projectile with a maximum yield of one molecule per incoming ion. In the same Fig. 4 (top panel) column density ratios of nitrogen oxides are given. As discussed in more detail below, the measurements of these ratios in astrophysical environments can give some hints on the presence and relative abundances of molecular oxygen and/or nitrogen. From the figure we can see that when mixed with water, NO is more than 10 times more abundant than $\mathrm{N}_{2} \mathrm{O}$ and about 2 times more abundant than $\mathrm{NO}_{2}$.

The $\mathrm{NO} / \mathrm{NO}_{2}$ ratio in $\mathrm{N}_{2}: \mathrm{O}_{2}$ mixtures is below $1 / 10$ and is not reported in Fig. 4.

In addition to these implantation experiments, which are more useful for application to ices in the solar system, we have conducted experiments by irradiating thin films (i.e. thinner than the penetration depth of used ions) mainly aimed at improving the astrophysical relevance of the results. Thin films in fact better represent icy mantles on grains in the interstellar medium that are irradiated by energetic cosmic ions. 
$\mathrm{Ph}$. Boduch et al.: Energetic ions induced chemistry

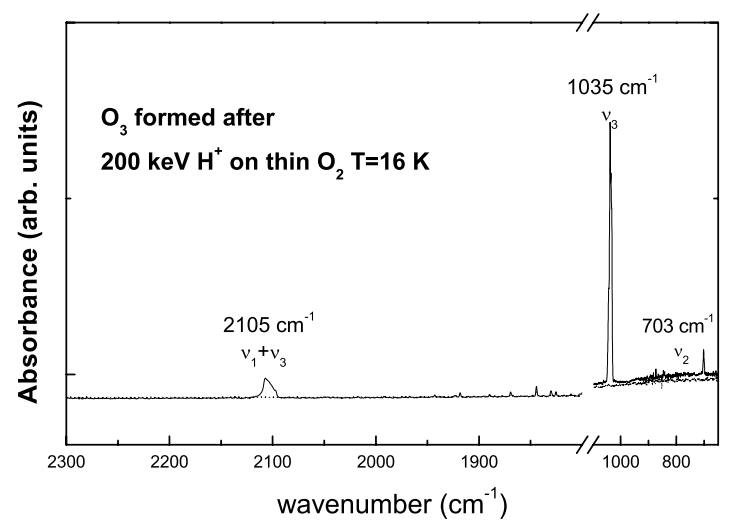

Fig. 5. Absorbance IR spectrum of a thin film of solid $\mathrm{O}_{2}$ at $16 \mathrm{~K}$ after bombardment by $200 \mathrm{keV} \mathrm{H}^{+}$ions. The dotted line is the featureless spectrum of the deposited oxygen target.

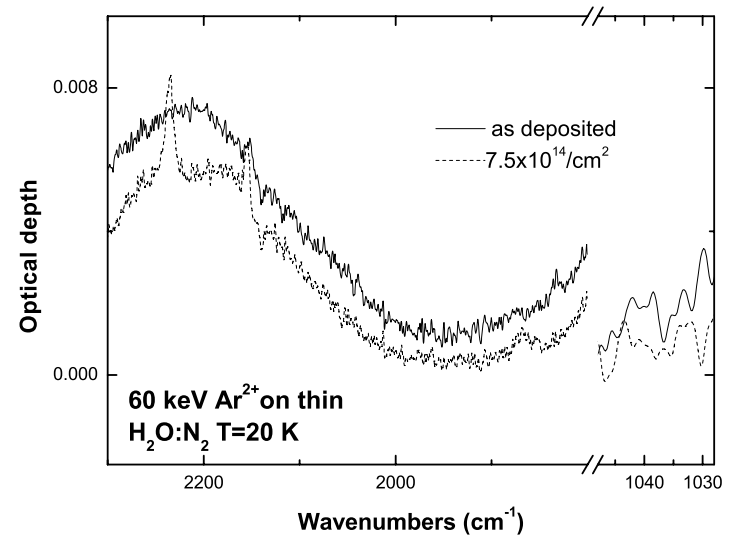

Fig. 6. IR spectra (in optical depth units) of a $\mathrm{H}_{2} \mathrm{O}: \mathrm{N}_{2}$ mixture as deposited at $20 \mathrm{~K}$ and after having been irradiated by $60 \mathrm{keV} \mathrm{Ar}^{2+}$ ions. The spectral range is the same of the spectra shown in Fig. 1 to favour a comparison.

In Fig. 5 the absorbance IR spectrum of a thin film of solid $\mathrm{O}_{2}$ at $16 \mathrm{~K}$ after bombardment by $200 \mathrm{keV} \mathrm{H}^{+}$ions is presented (the dotted line is the featureless spectrum of the deposited oxygen target). We confirm the formation of abundant ozone, whose spectrum was also used for its identification in the experiments discussed above.

Infrared spectra (in optical depth units) of a $\mathrm{H}_{2} \mathrm{O}: \mathrm{N}_{2}$ mixture as deposited at $20 \mathrm{~K}$ and after having been irradiated by $60 \mathrm{keV} \mathrm{Ar}^{2+}$ ions are presented in Fig. 6. The spectral range is the same as in the spectra shown in Fig. 1 to facilitate comparison. The formation of $\mathrm{N}_{2} \mathrm{O}$ is made clear by the observation of the $2234 \mathrm{~cm}^{-1}$ band. Although less abundant, both $\mathrm{NO}$ and $\mathrm{NO}_{2}$ are formed while $\mathrm{O}_{3}$ is not detectable.

\section{Astrophysical implications}

One of the purposes of this paper is to study the possibility to prove the presence of frozen $\mathrm{N}_{2}$ and $\mathrm{O}_{2}$ with indirect methods, i.e., by detecting molecular species formed by radiolysis of astrophysically relevant ice mixtures.

As a whole, the experiments have shown that both irradiated thin films and carbon-implanted thick films give similar results, except that the latter produce species containing the projectile. In the next paragraphs we suggest some possible consequences for the chemistry of ices in astrophysical environments. Of course there are alternative ways to produce nitrogen oxides in astrophysical environments. These include chemical reactions on cold grains in the interstellar medium and/or in the solar nebula at the time of the planetary accretion, photolysis and thermal processing. A comparison of the relevance of each of these formation routes is beyond the scope of the present paper, however.

\subsection{Ices in dense clouds}

As discussed above, $\mathrm{O}_{2}$ and $\mathrm{N}_{2}$ are expected to accrete onto icy grain mantles in dense molecular clouds. When these mantles are irradiated by cosmic ions, new species such as $\mathrm{O}_{3}, \mathrm{NO}$, $\mathrm{NO}_{2}$, and $\mathrm{N}_{2} \mathrm{O}$ will form. However, the identification of these solid phase species in astronomical IR spectra is not straightforward. The most intense band of ozone, at about $1038 \mathrm{~cm}^{-1}$, is superposed to the band due to silicates and is very close to the methanol band at about $1026 \mathrm{~cm}^{-1}$ and also to the ammonia band at about $1110 \mathrm{~cm}^{-1}$ (e.g. Bottinelli et al. 2010). The $\mathrm{N}_{2} \mathrm{O}$ and $\mathrm{NO}_{2}$ bands lie at about 2234 and $1605 \mathrm{~cm}^{-1}$, respectively, close to $\mathrm{CO}_{2}$ and $\mathrm{H}_{2} \mathrm{O}$ band regions. Keane et al. (2001) and Boogert et al. (2008) have performed a detailed analysis of the profile of the $5-8 \mu \mathrm{m}$ bands observed toward several high-mass and low-mass young stellar objects, respectively. The carriers of these bands have not been firmly identified yet. It is suggested that the bands' profile is caused by solid $\mathrm{H}_{2} \mathrm{O}$ and to five different components. Some molecular species, such as $\mathrm{CH}_{3} \mathrm{OH}$, $\mathrm{NH}_{3}, \mathrm{H}_{2} \mathrm{CO}, \mathrm{HCOOH}$, and $\mathrm{HCOO}^{-}$are expected to contribute to these absorption bands. Here, we suggest that $\mathrm{NO}_{2}$ could contribute to this absorption as well. However, more experimental work is necessary to confirm this hypothesis. The band generated by NO lies at about $1870 \mathrm{~cm}^{-1}$ (see Table 2). This band is not superposed to other strong bands generated by abundant solid phase species (e.g. Gibb et al. 2004) and could be searched for in IR astronomical spectra of dense molecular clouds.

However, NO is predicted by chemical models to be present on grains (Charnley et al. 2001) where, by addition of $\mathrm{H}$ atoms in a surface reaction scheme, it might produce hydrogenated species such as hydroxylamine $\left(\mathrm{NH}_{2} \mathrm{OH}\right)$. This prediction of chemical models has recently been confirmed by laboratory experiments of NO hydrogenation that demonstrated the destruction of $\mathrm{NO}$ and the formation of $\mathrm{HNO}, \mathrm{N}_{2} \mathrm{O}$ and $\mathrm{NH}_{2} \mathrm{OH}$ (Congiu et al. 2012). These authors showed that if hydrogenation dominates, $\mathrm{NO}$ is rapidly destroyed and $\mathrm{NH}_{3}, \mathrm{~N}_{2} \mathrm{O}$ and $\mathrm{NH}_{2} \mathrm{OH}$ are the most abundant N-bearing species (see Fig. 3 in Congiu et al. 2012).

Energetic processes such as those discussed here can be considered as de-hydrogenation processes that, in the present experiments, showed that $\mathrm{NO}$ is costantly more abundant than $\mathrm{N}_{2} \mathrm{O}$ and that no hydrogenated species (e.g. $\mathrm{NH}_{2} \mathrm{OH}$, or $\mathrm{NH}_{3}$ ) are produced. Thus the detection of nitrogen oxides in icy grain mantles and the measurement of their relative abundance could help to distinguish which process (hydrogenation vs energetic processing) is the most relevant mechanism to drive the chemistry of grains in dense molecular clouds.

\subsection{Ices in solar system objects}

The experimental results described here were obtained at low temperatures and are only marginally relevant to the icy moons of Jupiter which have a higher surface temperature $(80 \mathrm{~K}$ and more). At Saturn and beyond, the temperatures decrease and our results can better mimic the interaction of fast cosmic ions with icy surfaces.

As said above, ozone has been observed on Dione and Rhea (Noll et al. 1997) and its presence has been attributed 
to radiolysis of pure water in the hypothesis that $\mathrm{O}_{2}$ is formed first. Indeed $\mathrm{O}_{2}$ has been detected to form a tenuous atmosphere around the moons (Johnson et al. 2008). However, at present, there is no experimental evidence of the formation of appreciable quantity of ozone after ion bombardment of pure water ice. The present results confirm that ozone is produced only after addition in the ice of other O-bearing species such as $\mathrm{O}_{2}$ (Cooper et al. 2008; this paper), and $\mathrm{CO}_{2}$ (Boduch et al. 2011, and references therein). Because of the temperatures of the surfaces of the moons $(50-100 \mathrm{~K})$, we believe that $\mathrm{CO}_{2}$ is a better candidate as parent molecule of the observed ozone.

The Pluto/Charon system, Triton, and trans-Neptunian objects are bodies where the present results could have the greatest relevance. $\mathrm{N}_{2}$ is certainly present on Pluto (Owen et al. 1993; Douté et al. 1999) and Triton (Cruikshank et al. 1993), where it is mixed with other molecules, among which those bearing oxygen are $\mathrm{CO}, \mathrm{CO}_{2}$ (on Triton), and also $\mathrm{H}_{2} \mathrm{O}$, which is much less prominent on Pluto than on Triton (Grundy \& Buie 2002). $\mathrm{O}_{2}$ has not been observed or predicted to be present there. Radiolysis by cosmic ions (solar wind and galactic cosmic rays) is active on those objects. It has been estimated that the Pluto and Triton surfaces accumulate a dose of about $100 \mathrm{eV} / 16 \mathrm{u}$ in 4.6 billion years. At a depth of 1 meter, the dose is reduced to $30 \mathrm{eV} / 16 u$ (e.g. Hudson et al. 2008). Here doses represent the amount of energy (eV) delivered by cosmic ions per "small" (16u) target molecule. These doses are sufficient to alter the chemical composition. Based on the present results, $\mathrm{NO}, \mathrm{NO}_{2}$ and $\mathrm{N}_{2} \mathrm{O}$ are formed (note that nitrogen oxides are also formed after ion bombardment of mixtures containing nitrogen and carbon monoxide, Sicilia et al. 2012). We suggest here that the measurement of molecular ratios $\mathrm{NO} / \mathrm{NO}_{2}$ and $\mathrm{NO} / \mathrm{N}_{2} \mathrm{O}$ in the ice and/or in the tenuous atmospheres of those objects are useful to confirm the presence of nitrogen and reveal if oxygen is present as well. Because Triton is richer in oxygen-bearing species, the abundance ratios $\mathrm{NO} / \mathrm{NO}_{2}$ and $\mathrm{NO} / \mathrm{N}_{2} \mathrm{O}$ would be lower than on Pluto (Fig. 4).

\subsection{Ices in extra-solar objects?}

At the present, numerous ground-based and space observations resulted in a total of 767 identified exo-planets (May 22, 2012) ${ }^{2}$. A very important objective is now the observation and physico-chemical characterization of the atmospheres of these exo-planets. For this purpose, the Exoplanet Characterisation Observatory, EChO, has been proposed and has been selected by ESA as one of four medium-class mission candidates (Tinetti et al. 2011).

In the past years, the first spectra of exoplanets using transit transmission and emission spectroscopy have been obtained and molecular species (e.g. gaseous water, methane, carbon monoxide, and dioxide) have been detected (e.g., Tinetti et al. 2010). It is obvious to assume that many exo-planets and exo-satellites have ices on their surfaces or on grains in the atmospheres. The interaction with cosmic ion populations is expected to drive chemical modifications of those ices, which, in turn, influence the atmospheric chemistry. In particular, we have shown that ozone is a good indicator for the presence of molecular oxygen (or other species such as $\mathrm{CO}_{2}$ ), while nitrogen oxides are the best candidate to hint at the presence of nitrogen mixed with water or oxygen. When mixed with water, $\mathrm{NO}$ is more than 10 times more abundant than $\mathrm{N}_{2} \mathrm{O}$ and about 2 times more than $\mathrm{NO}_{2}$ (Fig. 4). It is trivial to say that many more experimental and observational

\footnotetext{
${ }^{2}$ http://exoplanet.eu/
}

data are essential to obtain a better understanding of the atmospheres and surfaces of these exo-planets with the ultimate objective to achieve insight into their evolution and habitability.

Acknowledgements. G.S. acknowledges the financial support given by European COST Action CM0805 (The Chemical Cosmos: Understanding Chemistry in Astronomical Environments) for his three-months visit at CIMAP-GANIL Labs. It is a pleasure to thank T. Been, S. Guillous, F. Noury, and J. M. Ramillon for their technical assistance.

\section{References}

Bergin, E. A., Alves, J., Huard, T., \& Lada, C. J. 2002, ApJ, 570, L101 Bergin, E. A., Melnick, G. J., Gerakines, P. A., Neufeld, D. A., \& Whittet, D. C. B. 2005, ApJ, 627, L33

Boduch, Ph., Frota da Silveira, E., Domaracka, A., et al. 2011, Adv. Astron., ID 327641, DOI: 10.1155/2011/327641

Boogert, A. C. A., Pontoppidan, K. M., Lahuis, F., et al. 2004, ApJ, 154, 359 Boogert, A. C. A., Pontoppidan, K. M., Knez, C., et al. 2008, ApJ, 678, 985 Bottinelli, S., Boogert, A. C., Bouwman, J., et al. 2010, ApJ, 718, 1100 Caselli, P., Walmsley, C. M., Zucconi, A., et al. 2002, ApJ, 565, 331 Charnley, S. B., Rodgers, S. D., \& Ehrenfreund, P. 1992, A\&A, 378, 1024 Congiu, E., Fedoseev, G., Ioppolo, S., et al. 2012, ApJ, 750, L12

Cooper, P. D., Moore, M. H., \& Hudson, R. L. 2008, Icarus, 194, 379 Cruikshank, D. P., Roush, T. L., Owen, T. C., et al. 1993, Science, 261, 742 Dalton, J. B., Cruikshank, D. P., Stephan, K., et al. 2010, Sp. Sci. Rev., 153, 113 Douté, S., Schmitt, B., Quirico, E., et al. 1999, Icarus, 142, 421

Ehrenfreund, P., Breukers, R., d'Hendecourt, L., \& Greenberg, J. M. 1992, A\&A, 260, 431

Foti, G., Calcagno, L., Sheng, K. L., \& Strazzulla, G. 1984, Nature, 310, 126 Fuchs, G. W., Cuppen, H. M., Ioppolo, S., et al. 2009, A\&A, 505, 629

Fulvio, D., Sivaraman, B., Baratta, G. A., Palumbo, M. E., \& Mason, N. J. 2009, Spectrochimica Acta, 72, 1007

Gerakines, P. A., Whittet, D. C. B., Ehrenfreund, P., et al. 1999, ApJ, 522, 357

Gibb, E. L., Whittet, D. C. B., Schutte, W. A., et al. 2000, ApJ, 536, 347

Grundy, W. M., \& Buie, M. W. 2002, Icarus, 157, 128

Hudson, R. L., Palumbo, M. E., Strazzulla, G., et al. 2008, Laboratory Studies of the Chemistry of Transneptunian Object Surface Materials, in The Solar System Beyond Neptune, eds. M. A. Barucci, H. Boehnhardt, D. P. Cruikshank, \& A. Morbidelli (Tucson: University of Arizona Press), 507

Jiang, G. J., Person, W. B., \& Brown, K. G. 1975, JChPh, 62, 1201

Johnson, R. E., Luhmann, J. G., \& Tokar, R. J. 2008, Icarus, 180, 393

Keane, J. V., Tielens, A. G. G. M., Boogert, A. C. A., Schutte, W. A., \& Whittet, D. C. B. 2001, A\&A, 376, 254

Loeffler, M. J., Baratta, G. A., Palumbo, M. E., Strazzulla, G., \& Baragiola, R. 2005, A\&A, 435, 587

Mennella, V., Palumbo, M. E., \& Baratta, G. A. 2004, ApJ, 615, 1073

Moore, M. H., Donn, B., Khanna, R., \& A'Hearn, M. F. 1983, Icarus, 16, 815

Noll, K. S., Roush, T. L., Cruikshank, D. P., Johnson, R. E., \& Pendleton, Y. J. 1997, Nature, 388, 45

Owen, T. C., Roush, T. L., Cruikshank, D. P., et al. 1993, Science, 261, 745

Palumbo, M. E. 2006, A\&A, 453, 903

Palumbo, M. E., \& Strazzulla, G. 1993, A\&A, 269, 568

Palumbo, M. E., Baratta, G. A., Brucato, J. R., et al. 1998, A\&A, 334, 247

Palumbo, M. E., Ferini, G., \& Baratta, G. A. 2004, AdSpR, 33, 49

Pilling, S., Seperuelo Duarte, E., Domaracka, A., et al. 2010, A\&A, 523, A77

Pugh L. A., \& Rao K. N. 1976, Molecular Spectroscopy: Modern research, Vol. III (New York: Academic Press)

Raut, U., Teolis, B. D., Loeffler, M. J., et al. 2007, J. Chem. Phys., 126, 244511

Sicilia, D., Ioppolo, S., Vindigni, T., Baratta, G. A. \& Palumbo, M. E. 2012, A\&A, 543, A155

Smith, M. A. H., Rinsland, C. P., Fridovich, B., \& Rao, K. N. 1985, Molecular Spectroscopy: Modern research, Vol. III (New York: Academic Press)

Smith, R. G., Sellgren, K., \& Tokunaga, A. T. 1989, ApJ, 344, 413

Spencer, J. R., \& Calvin, W. M. 2002, AJ, 124, 3400

Strazzulla, G. 2011, NIMB, 2011, 269, 842

Strazzulla, G., \& Baratta, G. A. 1992, A\&A, 266, 434

Strazzulla, G., Baratta, G. A., \& Palumbo, M. E. 2001, Spectrochim. Acta A, 57, 825

Tielens A. G. G. M., Tokunaga, A. T., Geballe, T. R., \& Baas, F. 1991, ApJ, 381, 181

Tinetti, G., Deroo, P., Swain, M. R., et al. 2010, ApJ, 712, 139

Tinetti, G., Beaulieu, J. P. Henning, T., et al. 2011, Exper. Astron. [arXiv: 1112.2728v1]

Whittet, D. C. B., Bode, M. F., Longmore, A. J., et al. 1988, MNRAS, 233, 321

Willner, S. P., Gillett, F. C., Herter, T. L., et al. 1982, ApJ, 253, 174

Yamada, H., \& Person, W. B. 1964, JChPh, 41, 2478 